\title{
Numerical Modelling of the Interaction Between Water Sprays and Hot Air Jets - Part II: Two-Phase Flow Simulations
}

\author{
Tarek Beji, Setareh Ebrahimzadeh, Georgios Maragkos, Bart Merci \\ Department of Flow, Heat and Combustion Mechanics, Ghent University-UGent, B-9000, \\ Ghent, Belgium
}

\begin{abstract}
The paper presents a comprehensive set of numerical simulations performed to examine the current Computational Fluid Dynamics (CFD) capabilities in the prediction of the interaction of a water mist spray with a vertical upward jet of hot air within an Eulerian-Lagrangian framework. The experimental tests considered herein are described by Zhou [Proceedings of the Combustion Institute, 2015]. The spray is a $30^{\circ}$ full cone water mist spray emerging from a nozzle that delivers a water flow rate of $0.084 \mathrm{lpm}$ at a pressure of $750 \mathrm{kPa}$. The vertical jet of hot air at $205^{\circ} \mathrm{C}$ is issued from a $72 \mathrm{~mm}$-diameter nozzle placed at $560 \mathrm{~mm}$ below the water spray nozzle. Three exit velocities of 3.3, 4.2 and $5.3 \mathrm{~m} / \mathrm{s}$ were examined. Gas phase simulations (described in the companion paper, Part I) have allowed to determine a set of parameters (e.g., cell size of $4 \mathrm{~mm}$ and modified Deardorff model for the turbulent viscosity) that are suitable for the water mist spray simulations. Moreover, it is shown here that a prescribed complex spray pattern with a full discharge angle of $60^{\circ}$ is required in order to match water spray profiles in the nozzle near-field. The three regimes of spray-jet interaction (i.e., water spray dominated, vertical jet dominated or equal influence of the spray and the vertical jet) are qualitatively well captured by the numerical simulations. However, the location of the interaction boundary is underestimated by up to $26 \%$. This could be partially attributed to modelling aspects related to, for example, turbulent dispersion or turbulence inflow conditions of the droplets. Uncertainties in the experimental
\end{abstract}


measurements must also be considered.

Keywords: CFD, Spray Dynamics, Spray-Jet Interaction

\section{Introduction}

Water sprays are known to be an efficient means for fire control and suppression. The interaction of a water spray system with a fire occurs at many levels. For instance, flames in direct contact with water (in sufficient amounts) are

5 extinguished. Another important aspect is spray surface cooling: when liquid droplets reach the surface of solid materials exposed to thermal radiation from the fire and to convective heat transfer from the hot gases, the surface temperature is kept low thanks to evaporative cooling; potential ignition is therefore inhibited [1. A third aspect is related to the interaction of a water spray with smoke. Typically, the induced smoke cooling and the entrainment of cool air into the water spray envelope cause a downward smoke displacement [2]. The hot gases can be confined in the immediate vicinity of the fire. The downward motion of the spray may also act as a water curtain [3] 4. If the water spray is applied directly above a smoke plume issued from a fire, the extent of the penetration of the former through the latter is an important parameter in assessing the level of fire control and suppression 5. The latter configuration, referred to in the literature as the spray-plume interaction, is the configuration of interest in this work.

The spray-plume interaction has been investigated experimentally in [6] for fires generated via heptane spray nozzles and with convective heat release rates of 0.5 MW, 1.0 MW and 1.5 MW. The sprinklers used were early suppression fast response (ESFR) sprinklers positioned at a ceiling clearance of $3 \mathrm{~m}$ from the fire source and delivering flow rates of $1.88 \mathrm{l} / \mathrm{s}$ up to $6.23 \mathrm{l} / \mathrm{s}$. The experimental data remained though limited to water accumulation measurements using buckets positioned at the level of the water source. These measurements are referred to as Actual Delivered Density (ADD) measurements and provide an estimate of the water flux that is actually penetrating the fire plume. In order to provide a 
more detailed characterization of the spray-plume interaction, Schwille et al. [7] carried out experiments in which 5,15 and $50 \mathrm{~kW}$ methane fires were exposed to a spray positioned at $1.5 \mathrm{~m}$ above and delivering flow rates that ranged from 6 to $106 \mathrm{~L} / \mathrm{min}$. More specifically, the extent of the interaction region has been associated with significant temperature fluctuations which are correlated with high levels of fluctuations in the infrared (IR) intensity. The position of maximum fluctuations was used as a measure of the location of the interaction between the fire and the spray. The quality of the experimental data in the spray-plume interaction scenario has been further improved by Zhou [8] who considered the case of an upward hot air jet (with vertical velocities of 3.3 to $5.4 \mathrm{~m} / \mathrm{s}$ and an initial temperature of $205^{\circ} \mathrm{C}$ ) and a water mist nozzle positioned at $0.560 \mathrm{~m}$ above and delivering a flow rate of $0.084 \mathrm{lpm}$. Detailed measurements were performed using laser-based particle image velocimetry (PIV) to acquire spatially-resolved velocity data and a shadow imaging system (SIS) to measure water droplet size and volume flux. Lately, Link et al. 9] made a remarkable effort in characterizing with more detail the initial spray structure in a spray-plume configuration using a spatially-resolved spray scanning system $(4 \mathrm{~S})$. The updraft from a real fire plume has been simulated by a forced air jet with a velocity of around 4 $\mathrm{m} / \mathrm{s}$ and at ambient temperature. Spray nozzles with k-factor $33.1 \mathrm{lpm} / \mathrm{bar}^{1 / 2}$ operating at 1.38 bar were mounted on a ceiling at $1.5 \mathrm{~m}$ above the air-jet outlet. One can clearly see from the four experimental programs described above and listed in a chronological order that experimental research on spray-plume interaction is more and more focused on more controllable conditions (e.g., from liquid and gas fires to hot and then cold air) at laboratory-scale and with more and more advanced diagnostics. Such controllable conditions might not reflect practical fire scenarios but the intent, as explicitly mentioned in [8] and [9], is to provide data for CFD validation. To the best of our knowledge, there is no well-established theory or semi-empirical correlations on the interaction between sprays and fire plumes given the very wide range of possible fire scenarios (e.g., in terms of power of the fire source or the characteristics of the sprinkler or the water mist nozzle). Thus, the computational fluid dynamics (CFD) tools 
are believed to be a very good way to deal with such a problem because they allow the study of a large number of cases and scenarios at reduced cost and may result in the development of generalized engineering correlations [5] and/or general design and installation rules [10]. The CFD studies undertaken in [11] and [12] remained though rather qualitative. For example, in [11], it is stated that the numerical results should be taken with great caution and that only after the models are validated can the (numerical) study be used as a design tool. It is in this context that we defined the aim of our work as a detailed assessment of a CFD tool, namely the Fire Dynamics Simulator (FDS), based on the experimental data displayed in [8]. We believe that we are only at the very beginning of the process of understanding the interaction of sprays and 70 plumes in the context of fire suppression, and validated CFD packages will play a very important role in the years to come to build up this knowledge and understanding, because with multiple CFD packages validated, the use of CFD as numerical experiments may become possible and reliable.

The experiments carried out in [8] have been indeed designed in order to provide detailed and high quality experimental data for the purpose of assessing, improving and, eventually, validating the current CFD capabilities in the prediction of two-phase flows for water/smoke interaction. More specifically, the configuration (addressed in this paper) consists of a ceiling-mounted water spray placed directly above the centre of a hot air jet issuing from a steel tube.

80 Prior to the water spray experiments, three experiments were performed for three hot air jets without a spray 8. The simulation of these tests has been carried out and described in 13 . It has been concluded that provided that a good set of modelling options (for example for the turbulent SGS viscosity model or turbulence inflow boundary conditions) is selected, the gas phase flow can be predicted with a relatively high level of accuracy. After characterizing the gas phase flow, the water spray was characterized in [8] in terms of droplet size and velocity and water volume flow rate at two different elevations from the nozzle (in the near-field and far-field of the spray) without hot air. Finally, the interaction of the three hot air plumes with the water spray was investigated 
through combined gas-liquid velocity and droplet size measurements.

In [14, numerical simulations of the experiments described above have been performed with the CFD code FireFOAM, which is based on the open source framework OpenFOAM. Contrarily to the so called isolated thermal plumes that were relatively well predicted (as confirmed later in [15]), the water spray tests were more difficult to predict in [14]. The simulations performed in [14] showed indeed that, in the near-field, the simulated spray is wider than the profile measured experimentally. Furthermore, there were substantial discrepancies in the predictions of the liquid volumetric flow rate and droplet velocity in the core of the spray envelope. Results for the far-field were more encouraging. The simulations of the spray-jet interaction cases revealed that the predicted penetration depths of the spray through the plumes are substantially overpredicted. These discrepancies can nevertheless be partially attributed to experimental difficulties in separating the gas phase and spray velocities [14. Additional FireFOAM simulations carried out in [16] have shown that increasing the discharge halfangle form from $15^{\circ}$ to $30^{\circ}$ (to better match the near-field spray pattern) does improve the results. However, the location of the interaction boundary remains underestimated.

The goal of this work is to improve the prediction of the penetration depth. The predictive capabilities of another CFD code are assessed based on the experiments described in [8]. This code is the Fire Dynamics Simulator (FDS 6) [17, 18.

\section{Experimental set-up}

\subsection{Nozzle parameters}

The nozzle used in [8] is a Delevan CT-1.5-30 ${ }^{\circ} \mathrm{B}$ full cone nozzle, which delivers a water flow rate of $0.08 \mathrm{lpm}$ at $690 \mathrm{kPa}$ with a $30^{\circ}$ initial spray angle. The water spray nozzle was actually operated in [8] at $\Delta P_{w}=750 \mathrm{kPa}$, delivering a water flow rate of $0.084 \mathrm{lpm}$. The nozzle diameter provided by the manufacturer is $D_{\text {nozzle }}=0.33 \mathrm{~mm}$. 


\subsection{Experimental configuration}

120

The nozzle is placed at $30 \mathrm{~mm}$ below a $1.220 \mathrm{~m} \times 1.220 \mathrm{~m}$ aluminium ceiling plate in a centred position. A vertical upward jet of hot air is issued from a 72 $\mathrm{mm}$ diameter steel tube at a fixed temperature of $205{ }^{\circ} \mathrm{C}$. Three air velocities were tested: $3.3,4.2$ and $5.3 \mathrm{~m} / \mathrm{s}$. Prior to spray/jet interaction experiments, air. More details are provided in 8 .

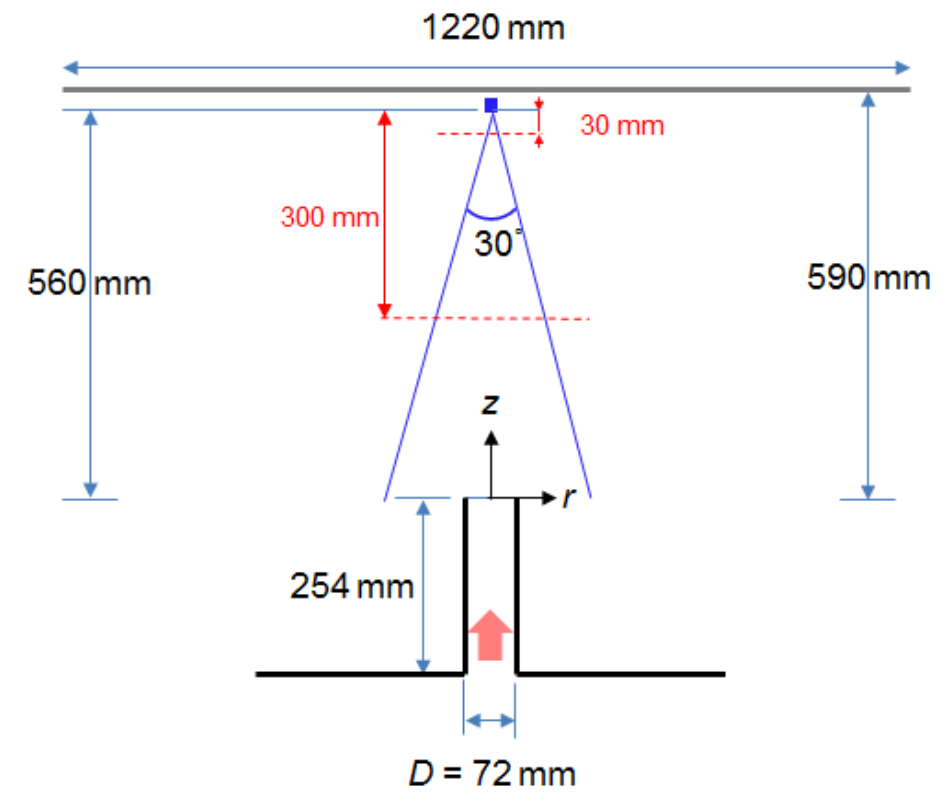

Figure 1: Schematic image of test configuration (not exactly up to scale).

\subsection{Instrumentation}

The water spray has been characterized at two distances from the nozzle (as shown in red in Fig. 1). Nozzle near-field and far-field measurements were performed at respectively $z=530 \mathrm{~mm}$ and $z=260 \mathrm{~mm}$ using a Shadow Imaging System (SIS) with high-resolution imaging and pulsed backlight illumination. These measurements consist of the (1) droplet size (in terms of volume-median 
diameter, VMD), (2) water volume flux, and (3) average droplet velocity. The minimum droplet size that could be detected was $12 \mu \mathrm{m}$.

The water volume flux is calculated as [19:

$$
\dot{V}_{d}^{\prime \prime}=\sum_{i=1}^{N} \frac{1}{6} \frac{\pi D_{d, i}^{3} u_{d, i}}{A \times d o f_{i}}
$$

where $D_{d, i}$ is the droplet diameter, $u_{d, i}$ is the droplet velocity, $A$ is the area of the field of the camera, dof is the depth of the field (which depends on the droplet size) and $N$ is the number of detected droplets.

The droplet average velocity, $\bar{u}_{d}$, is not calculated directly. First, the spray

140

volume density is calculated as [8, 19]:

$$
\alpha_{d}=\sum_{i=1}^{N} \frac{1}{6} \frac{\pi D_{d, i}^{3}}{A \times d o f_{i}}
$$

Then, $\bar{u}_{d}$ is calculated as $[8,19$ :

$$
\bar{u}_{d}=\frac{\dot{V}_{d}^{\prime \prime}}{\alpha_{d}}
$$

\section{Numerical modelling}

As mentioned above, the simulations described herein have been performed using FDS 6 (and more specifically FDS 6.5.3) [17, [18. The main aspects related to the gas phase modelling have been addressed in the companion paper [Numerical Modelling of the Interaction Between Water Sprays and Hot Air Jets - Part I: Gas Phase Large Eddy Simulations]. The focus will be put here on the spray modelling aspects. As reported in [6], the numerical modelling of a water spray can be divided into two categories depending on how the drops in the spray are traced. The gas flow is (typically) treated as an Eulerian coordinate system, but the liquid flow can be treated in either Eulerian or Lagrangian systems. In FDS 6, a Lagrangian approach is used for the liquid flow. The liquid water droplets are treated as Lagrangian particles that are injected near the nozzle and their trajectories within the computational domain are tracked, taking into account momentum and heat transfer with the surrounding gas phase. More details are provided hereafter. 


\subsection{Spray injection model}

Due to the limited ability to predict sprinkler atomization in CFD simulations, water droplets are introduced into the computational domain at a prescribed distance, $r_{0}$, from the nozzle, using an Eulerian-Lagrangian approach. As a result, the spray boundary consists of a spherical surface defined by $r_{0}$ and two angles, namely the elevation angle $\theta(\theta=0$ for the vertical direction) and the azimuthal angle $\varphi$. Droplets are assumed to emerge from the surface $r_{0}(\theta, \varphi)$ with an initial velocity calculated as [10]:

$$
u_{d, 0}=C \sqrt{\frac{\Delta P_{w}}{\rho_{d}}}
$$

where $\rho_{d}$ is the water density and $\Delta P_{w}$ the pressure at which the nozzle is operating. The constant $C$ is a factor that accounts for friction losses in the nozzle, estimated in [10] as $C=0.95$.

Furthermore, the water volume flux is uniformly distributed over $\varphi$ (i.e., full solid cone). The distribution over $\theta$ could either be uniform or follow a Gaussian distribution as [10, 18]:

$$
f_{v, \Omega}(\theta)=\exp \left[-\beta\left(\mu-\frac{\theta-\theta_{\text {inner }}}{\theta_{\text {outer }}-\theta_{\text {inner }}}\right)^{2}\right]
$$

where $\beta$ is a spread parameter (the default value in $[18$ is $\beta=5$ ) and $\mu$ is a parameter that gives the location in the spray at which most of the water is released. By default $\mu=0$, indicating that most of the water is released in the core region (i.e., axis) of the spray. The angles $\theta_{\text {inner }}$ and $\theta_{\text {outer }}$ delimit the inner and outer boundary of the spray.

Finally, the initial droplet size distribution of the water spray is expressed in terms of its Cumulative Volume Fraction (CVF) which is assumed here to fit a combination of the Rosin-Rammler with the lognormal function [20]:

$$
F_{v, D_{d}}\left(D_{d}\right)= \begin{cases}\frac{1}{\sigma \sqrt{2 \pi}} \int_{0}^{D_{d}} \frac{1}{\delta} \exp \left(-\frac{\left[\ln \left(D_{d}\right)-\ln \left(D_{v 50}\right)\right]^{2}}{2 \sigma^{2}}\right) d \delta & ; \quad D_{d} \leq D_{v 50} \\ 1-\exp \left[-\ln (2)\left(\frac{D_{d}}{D_{v 50}}\right)^{\gamma}\right] & ; \quad D_{d}>D_{v 50}\end{cases}
$$


where $D_{v 50}$ is the volume-median diameter (VMD), $\gamma$ is the spread factor and $\sigma$ is a standard deviation calculated as:

$$
\sigma=\frac{2}{\sqrt{2 \pi}(\ln (2)) \gamma}
$$

to ensure continuity at $D_{v 50}$. The VDM denotes that half of the cumulated water volume is represented by droplets having a diameter smaller than $D_{v 50}$.

\subsection{Lagrangian particle model}

The conservation equations of mass, momentum and energy are detailed in [17, 18. Droplet evaporation is calculated as a function of the droplet surface area, $A_{d}$, and a mass transfer coefficient, $h_{m}$, times the difference in the gas vapor fraction, $\tilde{Y}_{g}$, and the liquid equilibrium vapor mass fraction, $Y_{\ell}$, evaluated using the Clausius-Clapeyron equation. The drag coefficient used in the momentum equation accounts for drag reduction in the case of dense sprays. However, for the case that will be examined hereafter, the local droplet volume fraction did not exceed $10^{-3}$. Thus, the spray may be regarded as dilute and the drag reduction effect is negligible.

It is computationally prohibitive to track all the droplets discharged from the nozzle. Instead, the droplets are divided into several sets, each set represented by one single computed droplet. The number of real droplets represented by the single simulated droplet is calculated as:

$$
n=\frac{\dot{m}_{w}}{N_{p} \bar{m}_{d}}
$$

where $\dot{m}_{w}$ is the mass flow rate of water discharged at the nozzle, $N_{p}$ is the number of particles per second released in the computational domain, and $\bar{m}_{d}$ is the average mass of a droplet which can be calculated as:

$$
\bar{m}_{d}=\frac{4}{3} \pi \rho_{d} \int_{0}^{\infty} f_{N}(\delta)\left(\frac{\delta}{2}\right)^{3} d \delta
$$

The numerical study undertaken in 21] in order to characterize the spray using FDS has shown that a particle injection rate of $N_{p}=3 \times 10^{6} \mathrm{~s}^{-1}$ provides a good compromise between computational time and accuracy (the error induced by a low value of $N_{p}$ being proportional to $1 / \sqrt{N_{p}}$ ) for the case at hand. 


\subsection{Numerical post-processing of the liquid phase}

205 in FDS as:

$$
\dot{V}_{d}^{\prime \prime}=\frac{1}{t_{e}-t_{s}} \int_{t_{s}}^{t_{e}} \frac{\sum_{i=1}^{N_{p}} n_{i} \pi\left(D_{d, i}^{3} / 6\right) w_{d, i}}{V_{\text {sample }}} d t
$$

where $t_{s}$ and $t_{e}$ are respectively the start time and end time for the integration, $N_{p}$ is the number of computational droplets injected into the domain (i.e., particle injection rate), $n_{i}$ is the number of real droplets represented by the single simulated droplet $i, w_{d, i}$ is the droplet velocity in the vertical direction and $V_{\text {sample }}$ is a sampling volume in which droplets are collected. The sampling volume is taken here as a sphere with a radius of $10 \mathrm{~mm}$. A similar approach is used in the experiments and applied to the number of detected droplets [19], except that the sampling volume is taken as $V_{\text {sample }}=A \times \operatorname{dof}$ where $A$ is the area of the field of the camera and dof is referred to as the depth of field, a quantity which depends on the droplet size.

Similarly to the experimental procedure, the time-integrated average droplet velocity in the vertical direction is calculated as:

$$
\bar{w}_{d}=\frac{\dot{V}_{d}^{\prime \prime}}{\alpha_{d}}
$$

where the water volume concentration (or spray volume density) is calculated 220 as:

$$
\alpha_{d}=\frac{1}{t_{e}-t_{s}} \int_{t_{s}}^{t_{e}} \frac{\sum_{i=1}^{N_{p}} n_{i} \pi \frac{D_{d, i}^{3}}{6}}{V_{\text {sample }}} d t
$$

The VMD is not post-processed directly in FDS. It is calculated here by: (1) obtaining a histogram of the number of particles per bin (i.e., a range of droplet diameters), (2) calculating the corresponding cumulative volume fraction (CVF) and then (3) determining the diameter for which CVF $=0.5$. The width of bin ${ }_{225} i$ considered here is $\Delta D_{d, i}=D_{d, i}^{\max }-D_{d, i}^{\min }=1 \mu m$. The CVF for a given bin $i$ 
is calculated as:

$$
C V F_{i}=\frac{\sum_{j=1}^{i} N_{i}\left(\pi \bar{D}_{d, i}^{3} / 6\right)}{\sum_{j=1}^{N_{b i n}} N_{i}\left(\pi \bar{D}_{d, i}^{3} / 6\right)}
$$

where $N_{b i n}$ is the number of bins, $N_{i}$ is the number of droplets collected in each bin $i$, and $\bar{D}_{d, i}$ is the average droplet diameter in a bin. The latter is calculated as $\bar{D}_{d, i}=\left(D_{d, i}^{\max }+D_{d, i}^{\min }\right) / 2$.

\section{Spray characterization simulations}

\subsection{Previous results}

A series of numerical simulations have been carried out in 21 in order to characterize the water spray predictions in the absence of the vertical jet of hot air. More particularly, a detailed sensitivity analysis has been performed on $(i)$ the water volume flux probability density function to represent the water spray pattern (i.e., angular and droplet size distributions), (ii) the particle injection rate, $N_{p},($ iii $)$ the turbulent viscosity model, and (iv) the cell size. In this study, it has been recommended to use the lognormal-Rosin-Rammler distribution with a uniform angular distribution of the water volume flux and a particle injection rate of $N_{p}=3 \times 10^{6} \mathrm{~s}^{-1}$. For the gas phase, a cell size of $4 \mathrm{~mm}$ is to be used along with the modified Deardorff model for turbulent viscosity.

The results obtained in 21] were in relatively good agreement with the experimental data. There are nevertheless discrepancies which led us to perform additional simulations here, seeking for a better agreement in the spray characterization. The discrepancies in the simulations carried out in [21] are the following:

- The results in the near field (i.e., $z=530 \mathrm{~mm}$ ) were characterized by an overestimation of the water volume flux and the droplet velocity in the centreline. 
- Furthermore, in the near field, a droplet velocity top hat profile is predicted with a maximum centreline value about $25 \%$ higher than the measured experimental value. Reducing the factor $\mathrm{C}$ in Eq.(1) to 0.75 produced slightly better results but the top-hat profile remained.

- Another interesting point to raise with respect to the spray characterization simulations is the narrow predicted numerical profile of VMD in the near-field as observed also in [14. In fact, the experimental measurements of VMD at $z=530 \mathrm{~mm}$ suggest that the injection angle is significantly higher than the $30^{\circ}$ angle given in the description of the nozzle as reported in 8. It seems in fact to be between $60^{\circ}$ and $70^{\circ}$.

- In the near field (i.e., $z=260 \mathrm{~mm}$ ), there is a significant underestimation of the VMD in the core of the spray. This is suggested in 14 to be indicative of an underestimation of the evaporation rate.

\subsection{Set-up of new simulations}

The observations described above have urged us to perform additional simulations to characterize the spray (in addition to the detailed study undertaken in 21]) with the intent of producing better numerical results. In the first simulation performed here, the spray pattern is defined as 4 jets each characterized by a mass fraction of the total flow and an initial velocity. The spray angles and mass fraction of each jet are estimated from the measured water volume flux profiles in the near-field. The initial velocity of each jet is fine-tuned in order to produce a good agreement with the near-field droplet velocity profile. The characteristics of the 4 jets are displayed in Table 1. For the remainder of the paper, the approach used in 21] will be referred to as the 'simple spray pattern model $^{\prime}$ (SSPM) (with an initial velocity of $26 \mathrm{~m} / \mathrm{s}$ ) and the approach using 4 jets will be referred to as the 'complex spray pattern model' (CSPM).

As described in [21, the water spray characterization experiment has been modeled using a computational area which was open to flow on the sides and the bottom of the domain. The upper part of the domain has been modeled as a wall 
Table 1: Characteristics of the 4 jets used in the CSPM simulations of the water spray.

\begin{tabular}{|c|c|c|c|}
\hline & Half-angle $\left(^{\circ}\right)$ & Mass fraction $(\mathrm{kg} / \mathrm{kg})$ & Initial velocity $(\mathrm{m} / \mathrm{s})$ \\
\hline Jet 1 & $0-9$ & 0.44 & 25 \\
\hline Jet 2 & $9-18$ & 0.33 & 25 \\
\hline Jet 3 & $18-27$ & 0.16 & 16 \\
\hline Jet 4 & $27-30$ & 0.06 & 7 \\
\hline
\end{tabular}

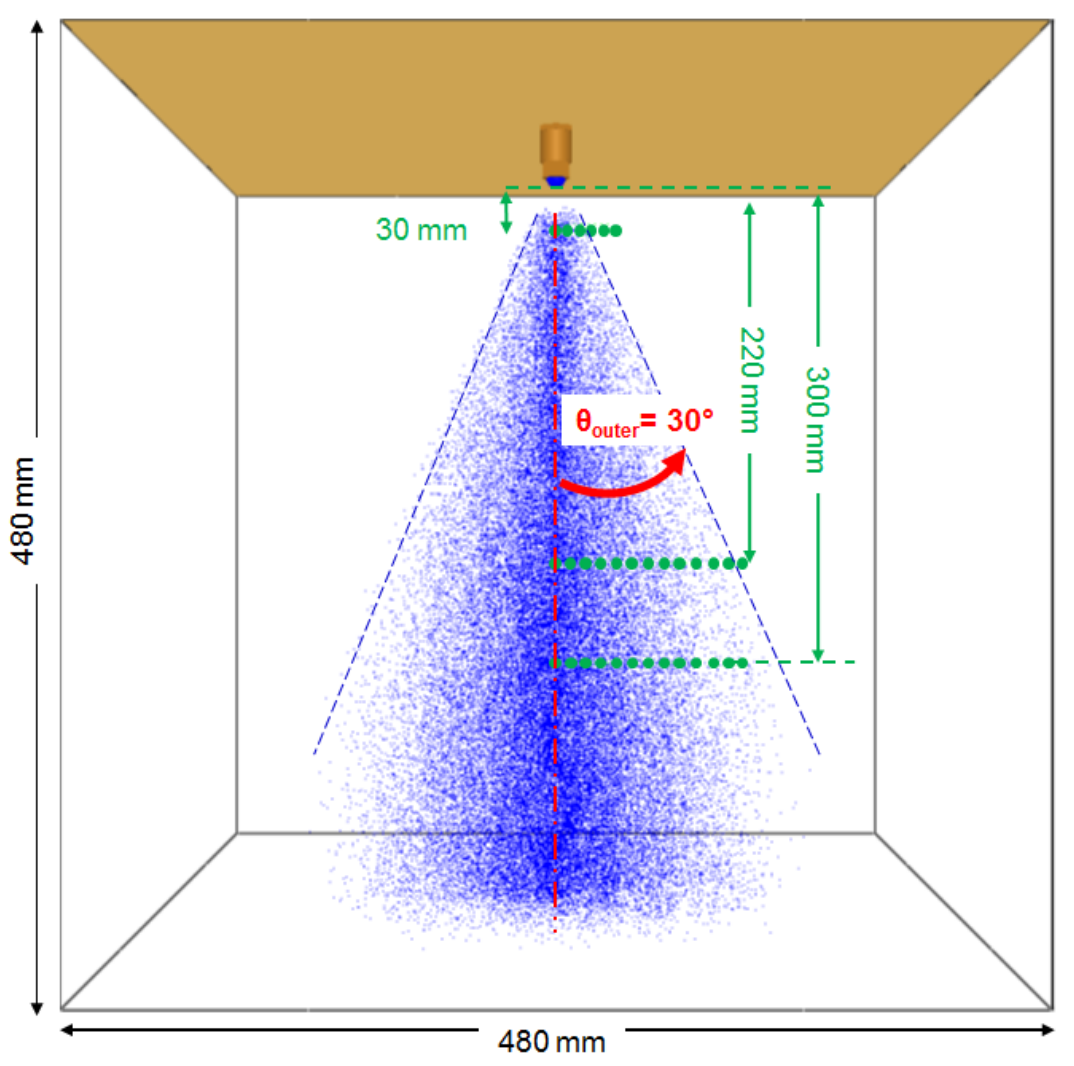

Figure 2: Visualization of the computational set-up (dimensions, position of the nozzle, location of the measurements (green dots) and water spray elevation angle) for the spray characterization simulations.

in order to represent the ceiling plate used in the experiments. The nozzle was placed at $30 \mathrm{~mm}$ from the ceiling. Note however, that the computational domain 
for the 'complex spray pattern model' has been extended to be $0.48 \mathrm{~m}$ high, 0.48 $\mathrm{m}$ wide and $0.48 \mathrm{~m}$ deep (as opposed to $0.4 \mathrm{~m}$ high, $0.2 \mathrm{~m}$ wide and $0.2 \mathrm{~m}$ deep) because the injection angle is wider and thus a wider domain is required to 

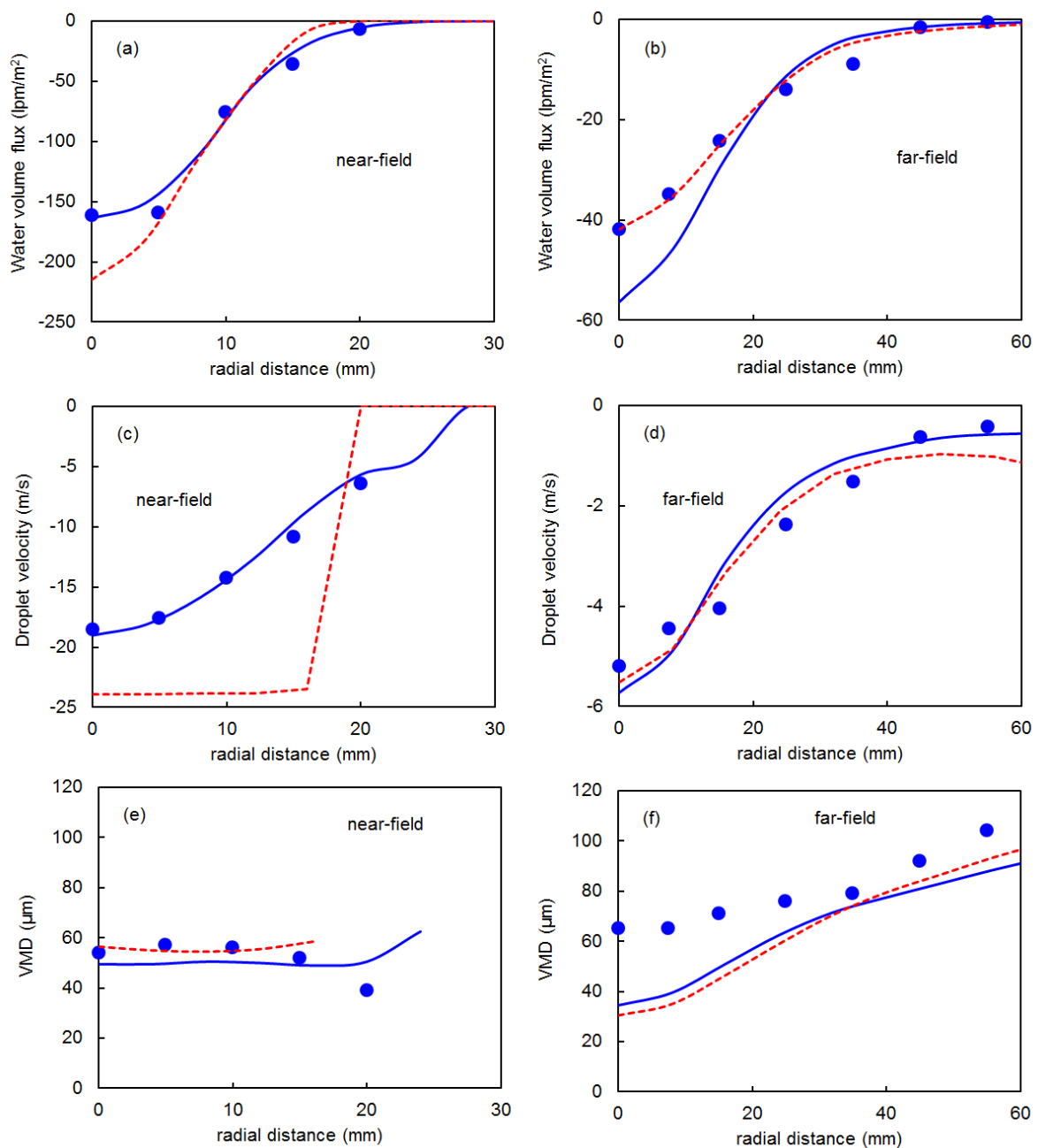

Figure 3: Comparison between measured and predicted water volume flux radial profiles in the near field $(z=530 \mathrm{~mm})$ and far field $(z=260 \mathrm{~mm})$ of the water spray only. The solid lines denote the CSPM simulation and the dashed lines denote the SSPM simulation

14] to explain the underestimation in VMD observed therein. Small droplets are easily entrained towards the core of the spray but do not evaporate, yielding both a low VMD and a high water volume flux.

The effect of lowering the temperature of the liquid water is first visualized in Fig.4 where lower values of water vapor concentration are observed in the CSPM (Evap-) simulation, which is indicative, as expected, of a reduced evap- 

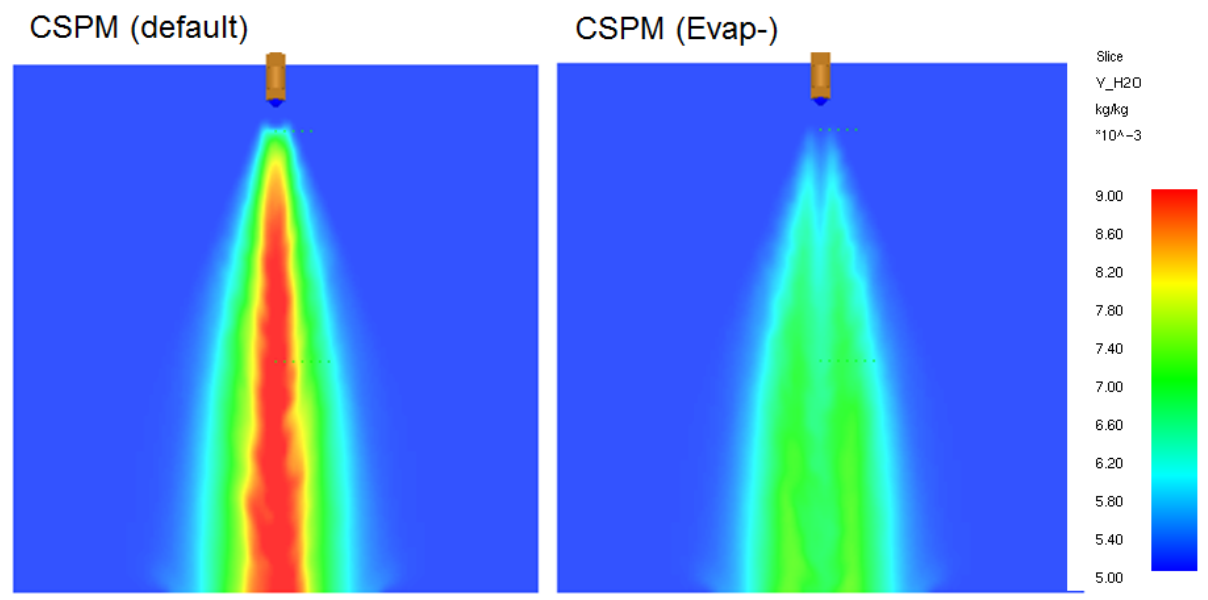

Figure 4: Influence of the liquid water temperature on the water vapor concentration.

oration rate. In the default simulation, i.e., CSPM (default), the steady-state evaporation rate is about $42 \mu \mathrm{g} / \mathrm{s}$, whereas in CSPM (Evap-) the evaporation rate is about $26 \mu \mathrm{g} / \mathrm{s}$. As observed in Fig.5, the effect of the $38 \%$ reduction in evaporation rate on the far-field profiles of the VMD and the water volume flux is negligible. This is due to the fact that the total amount of evaporated water is significantly (orders of magnitude) lower than the total amount of injected water. Nevertheless, as expected, the water volume fluxes increase with 325 a reduced evaporation rate.
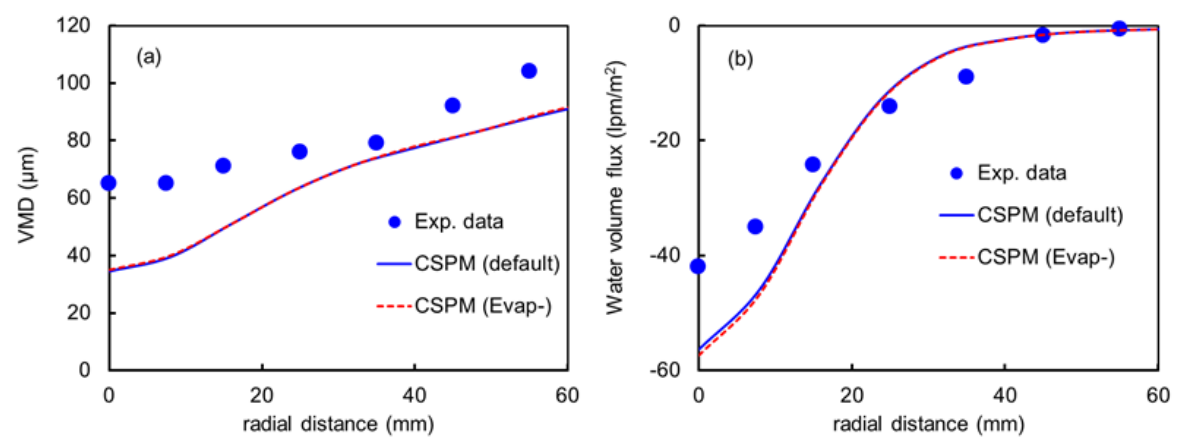

Figure 5: Influence of the liquid water temperature on the far-field profiles of (a) VMD and (b) water volume flux. 
In the light of these results for the spray simulation (without hot air), evaporation does not seem to be a key issue that might explain the lower VMD values in the centreline far-field. Furthermore, we examined the effect of the dynamic Smagorinsky model, which yielded slightly lower water volume fluxes in the spray core in the far-field but the VMD profile remained practically unchanged. The effect of the dynamic Smagorinsky model on the interaction cases is also negligible (not shown here). Other reasons, that are not explored in this paper, could be related to the droplet size distribution, the calculation of the drag coefficients or the effect of turbulent dispersion which could be significant, given the small size of the droplets involved in the calculations. Possible measurement errors, for example for the VMD, should also be considered.

\section{Spray-Jet interaction simulations}

\subsection{Set-up of the simulations}

Figure 6 shows the computational domain used in this study with the following dimensions: $0.720 \mathrm{~m} \times 0.720 \mathrm{~m} \times 0.592 \mathrm{~m}$ (hot air tube not included). Figure 6 shows 9 blocks of mesh for the main domain; an additional block is used for the inlet tube. Each of the 10 blocks is assigned to 1 processor. As mentioned earlier, a cell size of $4 \mathrm{~mm}$ is used along with the modified Deardorff model for the turbulent viscosity. The prescribed inlet velocities for the three cases are $3.10,3.95$ and $5.00 \mathrm{~m} / \mathrm{s}$. Turbulence inflow boundary conditions have been simulated using the SEM model by generating 1000 eddies of $6.4 \mathrm{~mm}$ that produce a velocity fluctuation of $13 \%$. Furthermore, in the spray-jet interaction simulations, water is discharged from $t=5 \mathrm{~s}$ onward in order to allow for the stabilization of the vertical jet. The data is averaged between 10 and $15 \mathrm{~s}$.

In addition to the hot air simulations, three simulations with cold air (and the same velocities) are carried out in order to examine the influence of the evaporation effect on the location of the interaction boundary. 
(a) Front view

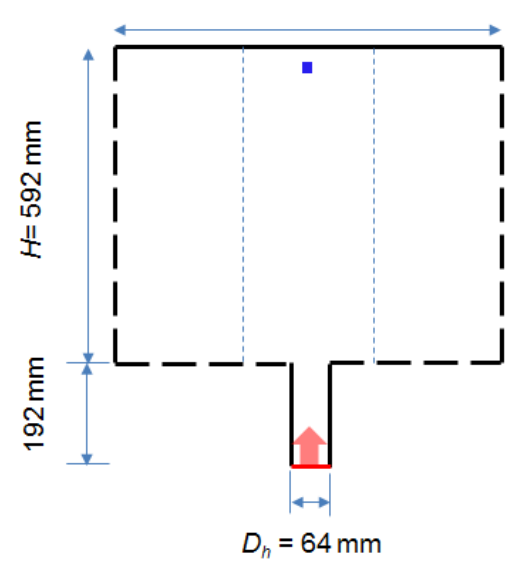

(b) Top view

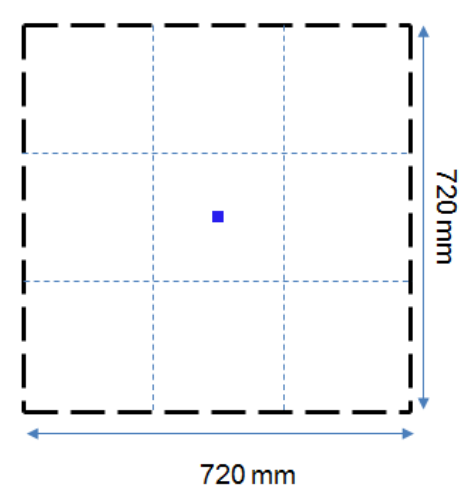

Figure 6: Computational domain for the spray-jet interaction simulations. The thick solid lines denote an INERT wall boundary condition. The dashed thick lines denote an OPEN boundary condition. The dashed thin lines denote the boundaries between several blocks of meshes used in this study.

\subsection{Results: location of the interaction boundary}

Figure 7 shows the predicted average gas vertical velocity flow field for the

three cases for respectively the simple spray pattern model and the complex spray pattern model. The water spray (in blue) clearly penetrates through the hot air jet (in red). The penetration height (solid black line), $z_{p}$ (also called interaction boundary), can be characterized by the height at which the mean centerline velocity is equal to zero.

The comparison of the numerical results obtained herein with the experimental data and the previous work of Meredith et al. 14 is provided in Table 2 which shows that the numerical results of Meredith et al. 14 have been improved by about $15 \%$ for cases 2 and 3 . This is mainly due to an increase in the set-up of the discharge half-angle from $15^{\circ}$ (as provided by the manufacturer) to $30^{\circ}$ to better match the experimental measurements of the water spray in the near-field. This has also been observed in [16] where the discharge half-angle has been set to $30^{\circ}$ instead of $15^{\circ}$. The improved prediction of the water spray in the near-field, using the complex spray pattern set-up, improved the predic- 
(a) simple spray pattern

vel. $(\mathrm{m} / \mathrm{s})$
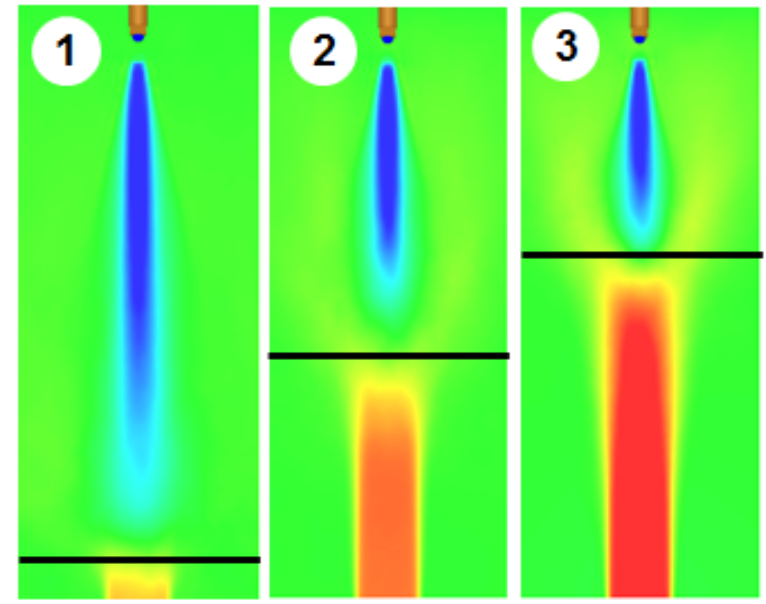

5.00

4.00

(b) complex spray pattern

0.00
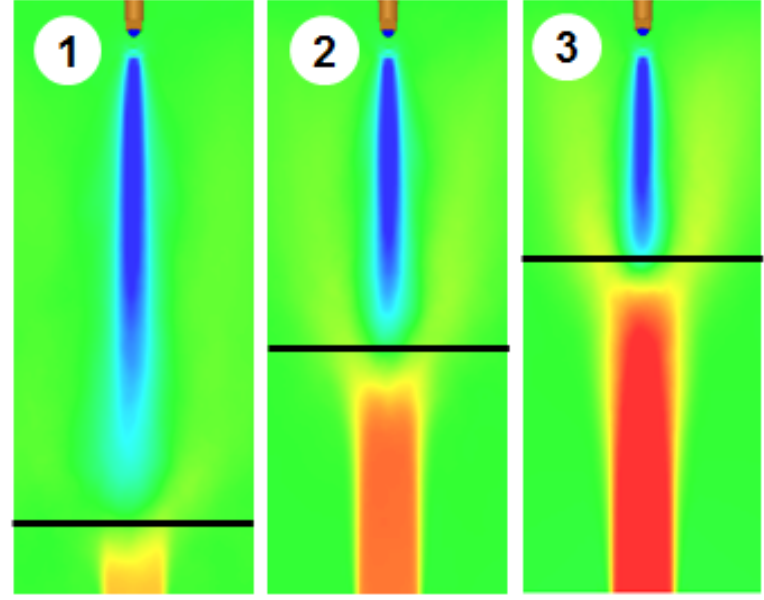

$-1.00$

2.00

1.00

3.00

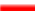

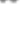

$-200$

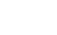

$-3.00$

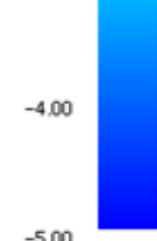

Figure 7: Predicted average gas vertical velocity flow field for the three cases.(a) Simple spray pattern model. (b) Complex spray pattern model. The location of the interaction boundary, $z_{p}$, is indicated with the solid black lines.

tion of the interaction boundary only for case 1 . The results for cases 2 and 3 remained almost unchanged.

Table 3 shows the results (in terms of interaction boundary location) for the cold air simulations which are compared to the hot air simulations. Except for 
Table 2: Measured and Predicted location of the interaction boundary, $z_{p}(\mathrm{~mm})$. The numbers between parenthesis correspond to the relative deviation between prediction and experimental data.

\begin{tabular}{|l|c|c|c|c|c|}
\hline Case ID & Exp. data & Pred.[14] & Pred.[16] & Pred. SSPM & Pred. CSPM \\
\hline Case 1 & 60 & $78(+30 \%)$ & $57(-5 \%)$ & $40(-33 \%)$ & $60(+0 \%)$ \\
\hline Case 2 & 320 & $193(-40 \%)$ & $240(-25 \%)$ & $240(-25 \%)$ & $240(-25 \%)$ \\
\hline Case 3 & 445 & $282(-37 \%)$ & $325(-27 \%)$ & $340(-24 \%)$ & $330(-26 \%)$ \\
\hline
\end{tabular}

case 1 , the predicted interaction layer is higher in the cold cases than in the hot cases. This is primarily due to the higher momentum of the air jet in the former cases (same velocities but higher density because of the lower temperature). It is believed that, for case 1 , the water evaporation effect prevails, leading to a higher interaction boundary in the hot case. These observations are in line with the findings in 8 , where it is stated that the interaction structure depends not only on momentum but on evaporation as well.

Table 3: Predicted location of the interaction boundary, $z_{p}(\mathrm{~mm})$, for the cold air simulations and comparison with the hot air simulations.

\begin{tabular}{|l|c|c|}
\hline Case ID & Hot air & Cold air \\
\hline Case 1 & 60 & 40 \\
\hline Case 2 & 240 & 280 \\
\hline Case 3 & 330 & 390 \\
\hline
\end{tabular}

The results displayed in tables 2 and 3 are expressed in terms of the interaction boundary (in $\mathrm{mm}$ or $\mathrm{m}$ ) as a function of the inlet velocity (in $\mathrm{m} / \mathrm{s}$ ). However, in order to be able to potentially scale-up the results for a sprayplume interaction configuration, it is best to use non-dimensional numbers. To this purpose, the interaction between a vertical jet and a spray is often examined in terms of ratio of momentum. In [6], if a sprinkler spray is described as a collection of water trajectories evenly divided between $\theta_{\text {inner }}=0^{\circ}$ and $\theta_{\text {outer }}$, 
then the trajectory with $\theta_{\text {outer }} / 2$ angle is taken as a representative of all the trajectories. Thus, the total momentum exerted by the spray can be approximated as [6]:

$$
M_{w}=\rho_{d} \dot{V}_{d}\left|u_{d, 0}\right| \cos \left(\theta_{\text {outer }} / 2\right)
$$

390 Inserting Eq.(4) into Eq.(14) gives:

$$
M_{w}=C \dot{V}_{d} \sqrt{\rho_{d} \Delta P_{w}} \cos \left(\theta_{\text {outer }} / 2\right)
$$

Using the values of $C=0.95, \dot{V}_{d}=0.084 \mathrm{lpm}, \rho_{d}=1000 \mathrm{~kg} / \mathrm{m}^{3}, \Delta P_{w}=750$ $\mathrm{kPa}$ and $\theta_{\text {outer }}=15^{\circ}$ for the water mist spray at hand gives a momentum of about $M_{w}=0.035 \mathrm{~N}$ (19\% lower than the value indicated in [8]).

The initial momentum of the hot air jet is calculated as:

$$
M_{a}=\int_{0}^{R} \rho_{0}(r) w_{0}^{2}(r) \pi r d r
$$

395

400 $\left(M_{a} / M_{w}\right)_{c a s e 2}=1.0$ and $\left(M_{a} / M_{w}\right)_{c a s e 3}=1.5$.

A non-dimensional penetration height (penetration ratio) is defined here as follows:

$$
z_{p}^{*}=\frac{\left(H-\ell_{n o z z l e}-z_{p}\right)}{H-\ell_{n o z z l e}}
$$

where $H$ is the ceiling height and $\ell_{\text {nozzle }}$ is the distance between the water injection height and the ceiling height $\left(\ell_{\text {nozzle }}=30 \mathrm{~mm}\right)$.

Figure 8 shows the experimental and numerical results for the non-dimensional penetration height for both the hot air and cold air configurations. Both experimental data and numerical predictions show a higher penetration ratio of the water spray when cold air is used. Furthermore, there is a noticeable improve- 


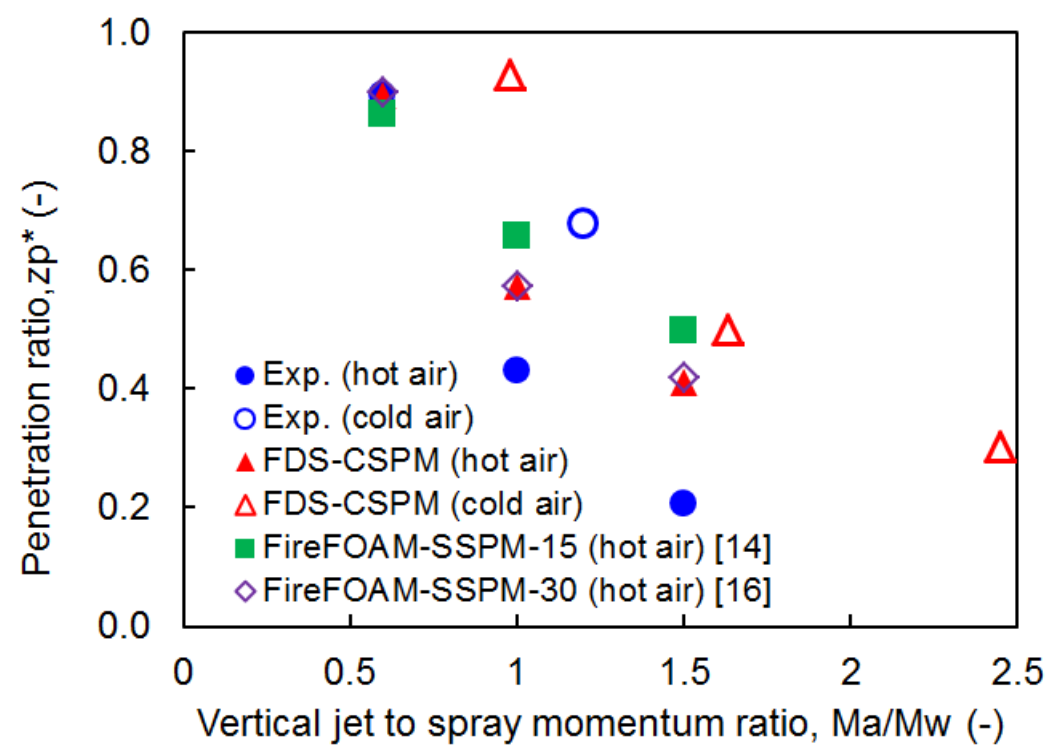

Figure 8: Comparison between the experimental and numerical non-dimensional penetration height, $z_{p}^{*}$, as a function of the vertical jet to spray momentum-ratio, $M_{a} / M_{w}$. Note that in 14 a discharge half-angle of $15^{\circ}$ has been prescribed along with a Gaussian distribution of the water droplets at the injection, whereas a half-angle of $30^{\circ}$ and a uniform distribution were prescribed in [16].

ment in the predictions in comparison to the results published in [14, but no significant differences with the results reported in [16].

\subsection{Results: heat absorption ratio}

In this study, we also examined the fraction of heat absorbed by the water droplets. Therefore, the hot air jets are also characterized in terms of convective heat release rates at the injection. The latter are calculated as follows:

$$
\dot{Q}_{c o n v}=\int_{0}^{R} \rho_{0}(r) c_{p} w_{0}(r)\left(T_{0}(r)-T_{a m b}\right) \pi r d r
$$

The convective heat release rates calculated in [8] are $\dot{Q}_{\text {conv,case } 1}=1.6 \mathrm{~kW}$, $\dot{Q}_{c o n v, c a s e 2}=2.1 \mathrm{~kW}$, and $\dot{Q}_{c o n v, c a s e 3}=2.6 \mathrm{~kW}$. 
The spray heat absorption ratio is defined here as:

$$
\dot{q}_{d}^{*}=\frac{\sum_{i=1}^{N} \dot{q}_{d, i}}{\dot{Q}_{c o n v}}
$$

where $N$ is the total number of droplets.

\subsection{Results: influence of the hot air on the water flux}

Figure 10 shows the influence of the hot air jet on the water volume flux radial profiles at $z=260 \mathrm{~mm}$. These results are in accordance with the results displayed in Fig. 7. The water volume flux at $z=260 \mathrm{~mm}$ is least affected in

at $z=z_{p}=40 \mathrm{~mm}$. Whereas in case 3 , all the water evaporated by $z=260$ $\mathrm{mm}$ (i.e., water volume flux equal to zero) because $z_{p}=340 \mathrm{~mm}>z=260$ $\mathrm{mm}$. Case 2 is an intermediate case. 


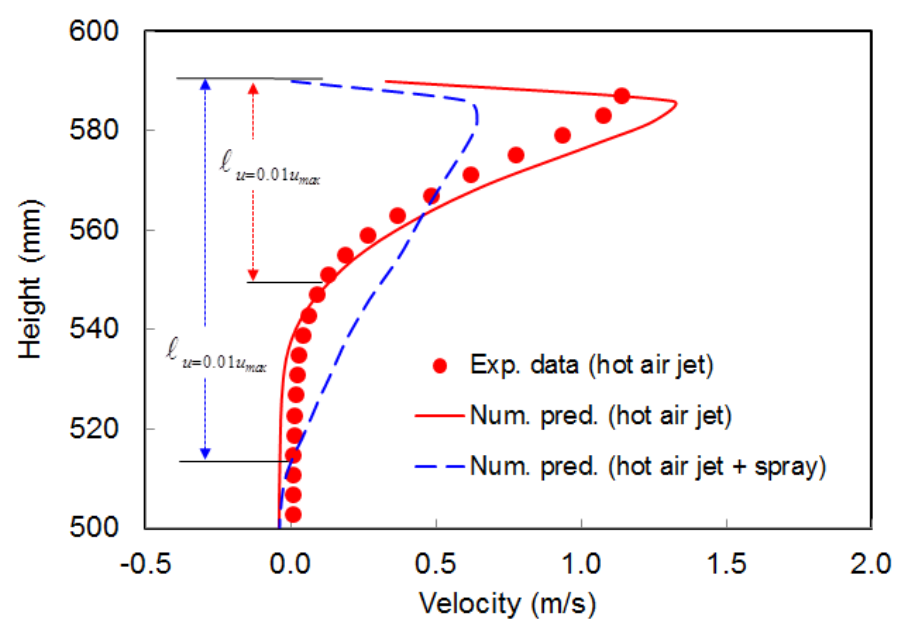

Figure 9: Predicted influence of the water spray on the ceiling-jet for case 2 at $r=310 \mathrm{~mm}$.

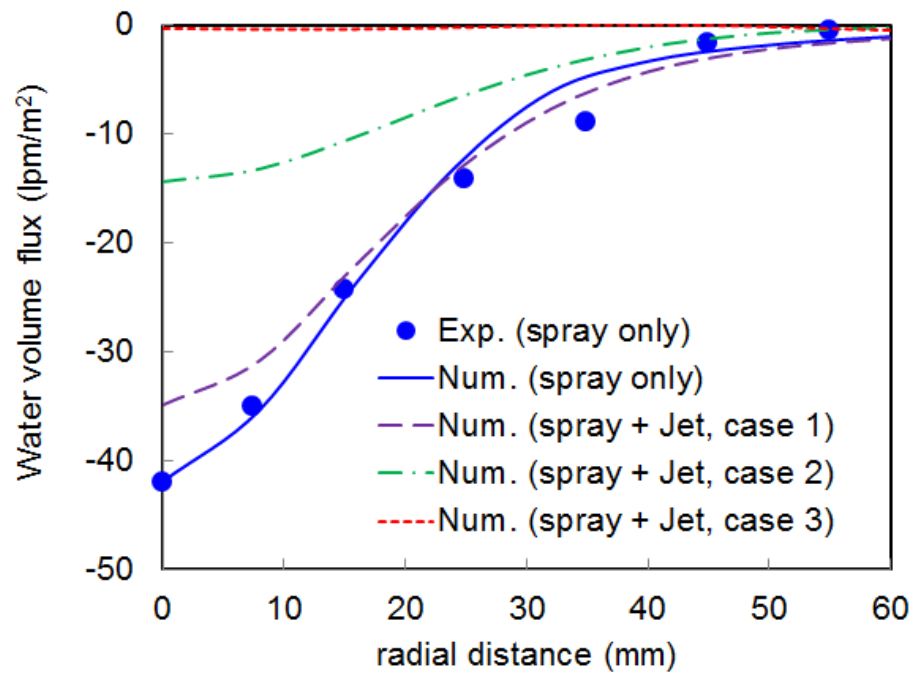

Figure 10: Influence of the hot air jet on the water volume flux radial profiles at $z=260 \mathrm{~mm}$.

\section{Conclusions}

The main objective of the companion paper (Part I) and this paper (Part II) is to assess the current capabilities of Computational Fluid Dynamics (CFD) in the prediction of a two-phase flow in a configuration of interest to fire suppres- 
sion. The scenario consists of a ceiling-mounted water mist nozzle positioned above a vertical jet of hot air that has been studied experimentally in 8 . The interaction of the water spray with the vertical jet is studied (among other aspects) in terms of penetration level of the water spray into the vertical jet of hot air.

In the experimental program carried out in $[8$, three campaigns were undertaken: $(i)$ impinging vertical jet on a horizontal ceiling plate, (ii) characterization of water spray only, and (iii) spray-jet interaction. After assessing the gas-phase simulations in Part I, we devoted this paper (Part II) to (ii) and (iii).

The spray characterization simulations carried out in 21] were essential in determining the influence of gas phase model settings (e.g., cell size, turbulent viscosity model) as well as liquid phase model settings (e.g., water flux angular distribution and particle injection rate, $N_{p}$ ). Note that the gas phase parameters (e.g., $D / \Delta x=18$ and modified Deardorff model for turbulent viscosity) are coherent with the findings of Part I. In this paper, the spray characterization simulations have been improved by using a complex spray pattern to describe water injection near the nozzle. Instead of injecting all the water droplets with the same initial velocity, the latter has been fine-tuned with respect to the discharge angle in order to reach a better agreement in the near-field profiles of water volume flux and droplet velocity. Evaporation effects have been examined to potentially explain discrepancies in the far-field profiles but the results were not conclusive.

470

The three regime of spray-jet interaction (i.e., water spray dominated, vertical jet dominated or equal influence of the spray and the vertical jet) are qualitatively well captured by the numerical simulations. However, the location of the interaction boundary is underestimated by up to $26 \%$. This could be partially attributed to modelling aspects related to, for example, turbulent 475 dispersion or turbulence inflow conditions of the droplets. Uncertainties in the experimental measurements must also be considered. 


\section{Acknowledgments}

Tarek Beji is a Postdoctoral Fellow of the Fund of Scientific ResearchFlanders (Belgium) (FWO Vlaanderen).

\section{References}

[1] A. Marshall, M. D. Marzo, Modelling aspects of sprinkler spray dynamics in fires, Process Safety and Environmental Protection 82 (2004) 97-104. doi:http://dx.doi.org/10.1205/095758204322972744

[2] Z. Tang, Z. Fang, J. Yuan, B. Merci, Experimental study of the downward displacement of fire-induced smoke by water sprays, Fire Safety Journal 55 (2013) 35-49. doi:http://dx.doi.org/10.1016/j.firesaf.2012. 10.014

[3] N. Hoffmann, E. Galea, An extension of the fire-field modelling technique to include fire-sprinkler interaction - i. the mathematical basis, International Journal of Heat and Mass Transfer 36 (1993) 1435-1444. doi:http://dx. doi.org/10.1016/s0017-9310(05)80054-7

[4] N. Hoffmann, E. Galea, An extension of the fire-field modelling technique to include fire-sprinkler interaction - ii. the simulations, International Journal of Heat and Mass Transfer 36 (1993) 1445-1457. doi:http://dx.doi.org/ 10.1016/s0017-9310(05)80055-9.

[5] R. Alpert, Numerical modelling of the interaction between automatic sprinkler sprays and fire plumes, Fire Safety Journal 9 (1985) 157-163. doi:http://dx.doi.org/10.1016/0379-7112(85)90003-7.

[6] S. Nam, Development of a computational model simulating the interaction 500 between a fire plume and a sprinkler spray, Fire Safety Journal 26 (1996) 1-33. doi:http://dx.doi.org/10.1016/0379-7112(96)00015-x. 
[7] J. Schwille, R. Lueptow, The reaction of a fire plume to a droplet spray,

Fire Safety Journal 41 (2006) 390-398. doi:https://doi.org/10.1016/ j.firesaf.2006.02.005.

[8] X. Zhou, Characterization of interactions between hot air plumes and water sprays for sprinkler protection, Proceedings of the Combustion Institute 35 (2015) 2723-2729. doi:http://dx.doi.org/10.1016/j.proci.2014.05. 078 .

[9] E. Link, T. Myers, A. Trouve, A. Marshall, Measurements of spray-plume interactions for model validation, Fire Safety Journal 91 (2017) 714-722. doi:https://doi.org/10.1016/j.firesaf.2017.04.024

[10] T. Sikanen, J. Vaar, S. Hostikka, A. Paajanen, Modeling and simulation of high pressure water mist systems, Fire Technology 50 (2014) 483-504. doi:http://dx.doi.org/10.1007/s10694-013-0335-8.

515 [11] S. Nam, Numerical simulation of the penetration capability of sprinkler 口 sprays, Fire Safety Journal 32 (1999) 307-329. doi:https://doi.org/10. 1016/s0379-7112(99)00004-1.

[12] J. Hua, K. Kumar, B. Kho, H. Xue, A numerical study of the interaction of water spray with a fire plume, Fire Safety Journal 37 (2002) 631-657. doi :https://doi.org/10.1016/s0379-7112(02)00026-7.

[13] T. Beji, G. Maragkos, S. E. Zadeh, B. Merci, Numerical modelling of the interaction between water sprays and hot air jets - part i: Gas phase a large eddy simulations, Fire Safety Journal 95 (2018) 77-86. doi:https: //doi.org/10.1016/j.firesaf.2017.11.005.

[14] K. Meredith, X. Zhou, S. Ebrahimzadeh, B. Merci, Numerical simulation of spray-plume interactions, 9th U.S. National Combustion Meeting.

[15] S. Ebrahimzadeh, G. Maragkos, T. Beji, B. Merci, Large eddy simulations of the ceiling jet induced by the impingement of a turbulent air plume, 
Fire Technology 52 (2016) 2093-2115. doi:https://doi.org/10.1007/ s10694-015-0561-3.

[16] S. Ebrahimzadeh, G. Maragkos, T. Beji, B. Merci, Large eddy simulations of water spray-hot air jet plume interactions, 10th Mediterranean Combustion Symposium (MCS10), Naples, Italy.

[17] K. McGrattan, S. Hostikka, R. McDermott, J. Floyd, C. Weinschenk, K. Overholt, Fire dynamics simulator, technical reference guide, volume 1: Mathematical model, National Institute of Standards and Technology, Gaithersburg, MD NIST Special Publication 1019 6th Edition (2017) 151 p.

[18] K. McGrattan, S. Hostikka, R. McDermott, J. Floyd, C. Weinschenk, K. Overholt, Fire dynamics simulator, user guide, National Institute of Standards and Technology, Gaithersburg, MD NIST Special Publication 1019 6th Edition (2017) 296 p.

[19] X. Zhou, S. DAniello, H.-Z. You, Spray characterization measurements of

■ a pendent fire sprinkler, Fire Safety Journal 54 (2012) 36-48. doi:http: //dx.doi.org/10.1016/j.firesaf.2012.07.007

[20] T. Myers, A. Marshall, A description of the initial fire sprinkler spray, घ Fire Safety Journal 84 (2016) 1-7. doi:http://dx.doi.org/10.1016/j. firesaf.2016.05.004.

[21] T. Beji, S. E. Zadeh, G. Maragkos, B. Merci, Influence of the particle injection rate, droplet size distribution and volume flux angular distribution on the results and computational time of water spray cfd simulations, Fire Safety Journal 91 (2017) 586-595. doi:https://doi.org/10.1016/ j.firesaf.2017.03.040. 